\title{
Performance Evaluation of bi-directional Roller Bearings for Seismic Isolation of Buildings Under Near-fault Earthquakes
}

Nelson Ortiz-Cano ( $\square$ nelson.ortiz@unimilitar.edu.co)

Universidad Militar Nueva Granada

Ricardo González-Olaya

Universidad Militar Nueva Granada

Carlos Gaviria-Mendoza

Universidad Militar Nueva Granada

Carlos Magluta

Universidade Federal do Rio de Janeiro Centro de Tecnologia

Ney Roitman

Universidade Federal do Rio de Janeiro Centro de Tecnologia

\section{Research Article}

Keywords: Seismic isolation, Roller isolation systems, Bi-directional roller bearings

Posted Date: August 24th, 2021

DOI: https://doi.org/10.21203/rs.3.rs-834301/v1

License: (9) This work is licensed under a Creative Commons Attribution 4.0 International License. Read Full License 


\title{
Performance evaluation of bi-directional roller bearings for seismic isolation of buildings under near-fault earthquakes
}

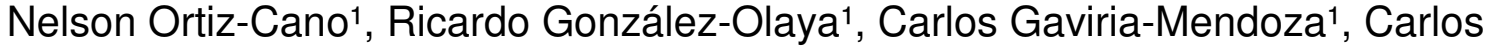 \\ Magluta ${ }^{2}$ and Ney Roitman².
}

${ }^{1}$ Department of Civil Engineering, Universidad Militar Nueva Granada, Cajicá, CU 250247, Colombia.

${ }^{2}$ Department of Civil Engineering, COPPE/UFRJ Centro de Tecnologia, Rio de Janeiro, CEP 21945-970, Brazil.

\begin{abstract}
An advantage bidirectional sloped rolling type isolation device composed of multiple rollers in both orthogonal-in-plane directions is studied in this research. The analytical model of a single direction of roller bearing (RB) system is extended to a two-direction RB system. Also, a 3D linear-elastic frame element to build the finite element model is used to incorporate the response of the building model. Several experimental tests of a physical building model with and without an RB system are used to validate the numerical model. The model is used to assess the nonlinear response history analysis of a four-story multi-column building system with two different physical properties that represent buildings with low and high lateral stiffness when subjected to pairs of scaled near-fault earthquake records. The effect of the angle of inclination of bearing plates in the range of $1.0^{\circ}$ to $4.0^{\circ}$ and sliding friction force is also investigated in a parametric analysis to evaluate the performance of RB with supplementary damping mechanisms ranging from 0.0 to $0.5 \mathrm{~N} / \mathrm{kg}$, i.e., friction force normalized with the structure mass. Results show that the proposed bi-directional RB system is suitable for reducing the seismic response of rigid and flexible multi-column structures. In particular, the RB system reduces structure acceleration responses by $5 \%$ to $85 \%$ in the flexible structure and $86 \%$ to $96 \%$ in the rigid structure. Furthermore, an angle of inclination of bearing plates greater than or equal to $3.0^{\circ}$ is an advantage to ensure the self-centering capacity.
\end{abstract}

Keywords: Seismic isolation, Roller isolation systems, Bi-directional roller bearings.

\section{Introduction}

Isolation systems have been successfully used since 1969 in 12.720 projects around the world Walters (2015). Although the early applications of this seismic protection mechanism were related to critical buildings such as hospitals and 
emergency facilities, more recently it has been extended to ensure better seismic response of lower and middle high residential-building projects (Wang et al., 2017).

New construction projects, updating of structures to current regulations, and the retrofitting of structures with insufficient resistance to withstand earthquakes are some examples in which this technology have been implemented (e.g., Naeim and Kelly, 1999; Tsai et al., 2007; Matsagar and Jangid, 2008; Hosseini and Soroor, 2011, 2013; Erdik et al., 2018; Ryan et al., 2018). Nevertheless, generalized applications of these devices are a current challenge that requires an enhancement of existing methods, alternative cost-effective devices, and other isolation strategies (Bagerzadeh-Karimi and Geneş, 2019; Calhoun et al., 2019; Beirami-Shahabi et al., 2020; Zhang and Ali, 2021).

Several studies have found that rolling based isolation developed excessive displacements under near-fault ground records (Rawat et al., 2018), stress concentration and lower friction resistance (Beirami-Shahabi et al., 2020), and more simple RB devices do not offer an integrated control of displacement (Zhang and Ali, 2021). These issues can be diminished by adopting energy- dissipation devices (Ortiz-Cano et al., 2015), e.g., implementation of shock absorbers with a particular bumper and gap configuration (Andreaus and De Angelis, 2020) or introducing traditional springs (Zhang and Ali, 2021).

Numerous attempts have been made to employ RB devices with a low value of friction force to cover a wide range of earthquake intensities, but with unnecessary high isolation level displacements as a result (Rawat et al., 2018; Rawat and Matsagar, 2021). Period-matching effects can arise on RB systems (i.e., tuned to the natural period of the building) even when located on lower floors (Harvey-Jr and Gavin, 2015). In addition, ground motions with long-period components that reach the natural period of the isolator device may weaken its behavior (Calhoun, 2018; Chen et al., 2021). In this sense, the robustness of RB isolators may be enhanced by a tailored design based on the seismic demand characteristics, i.e., period and intensity of ground motion (Harvey-Jr and Gavin, 2015).

Sliding friction mechanisms are (commonly) integrated into the RB system to provide additional friction force as reducing its peak displacement (Lee et al., 2010). The coefficient of friction between surfaces will deteriorate over time because of wear during recurrent loading cycles and weathering from ex- ternal conditions (Lee et al. 2010), and sliding velocity, surface temperature, and other physical conditions (Lee et al., 2010; Zhang and Ali, 2021). In this direction, one of the primary research areas is to develop reliable analytical and numerical models for predicting the behavior of the isolation system (Beirami-Shahabi et al., 2020). Also, an experimental calibrated model of a real-scaled implemented isolation device with particular dimensions (e.g., elastic sliding bearing) has been shown to reproduce its behavior under other ground motion but it is restricted to the device to which it was 
adjusted (Brewick et al., 2020). Thus, returning to RB devices, a reasonable range of constant slope angles and friction forces might be recognized as benchmarks in the foreseeable future (Wang et al., 2020).

In this paper, an advantaged bidirectional sloped rolling-type isolation device composed of multiple rollers in both orthogonal plane direction and pounding prevention enhancement is studied. First, an extension of the previous model developed by Ortiz-Cano et al. (2015) to couple the bidirectional nonlinear responses of RB system subject to a pair of horizontal ground motions components is presented. The nonlinear time history analysis of building with the proposed RB system is performed in The MathWorks Inc. (2019). Several experimental tests of a scaled building model, RB system, and the scaled building model with the RB system are analyzed. Then, the performance of a lower and median-rise building with RB system subjected to near-fault ground motions are numerically studied for several sloping angles of the bearing plate and sliding friction forces. Finally, some remarks and recommendations are stated.

\section{Dynamic behavior of isolated buildings with RB system}

Ortiz-Cano et al. (2015) studied a sloped rolling-type isolation device in which multiple rollers move between a $\mathrm{V}$-shaped bearing plate and a flat surface in one horizontal direction. The dynamic behavior of isolated buildings with RB systems under base excitations in a multiple degrees of freedom (MDoF) system in time domain is given in Equation 1,

$$
M \ddot{u}+C \dot{u}+K u+R\left(f_{s}+f_{d r}+f_{d s}\right)=-M \Gamma \ddot{u}_{g}
$$

Where $M, C$ and $K$ are the mass, damping and stiffness matrices of the MDoF system, respectively. $\ddot{u}, \dot{u}$ and $u$ are the acceleration, velocity, and displacement vectors.

On the other side, in Eq.1, $\ddot{u}_{g}$ is the seismic excitation vector that contains the base accelerations for each direction in which the building can be excited, i.e., 3 translational and 3 rotational DoF's in the Cartesian space. The term $\Gamma$ is an influence matrix that relates the excited DoF's $i$ with the direction of the seismic excitation $j$. The elements of the $\Gamma$ matrix take values of 0 or 1 according to the following expression:

$$
\Gamma_{i j}=\left\{\begin{array}{lc}
0 & \text { if DoF } i \text { isn't excited in } j \text { direction } \\
1 & \text { if DoF } i \text { is excited in } j \text { direction }
\end{array}\right.
$$

where, $R$ is a matrix that allocates the forces of restoration $f_{s}$, rolling friction $f_{d r}$ and sliding friction $f_{d s}$ developed by the RB system in $j$ direction into a matrix that contains the forces in the DoF's system in i direction. The elements that make up the $R$ vector take values of 0 or 1 as follows 


$$
R_{i j}=\left\{\begin{array}{rr}
0 & \text { if in DoF } i \text { the forces } f_{s}, f_{d r} \text { and } f_{d s} \text { aren't developed in } j \text { direction } \\
1 & \text { if in DoF } i \text { the forces } f_{s}, f_{d r} \text { and } f_{d s} \text { aren developed in } j \text { direction }
\end{array}\right.
$$

In this study, the forces $f_{s}, f_{d r}$ and $f_{d s}$, which characterize the RB system along one horizontal motion path, are extended for two orthogonal directions. These forces are defined in tensorial notation through Equations 4, 5, 6 and 7 as follows,

$$
\begin{aligned}
& f_{s}^{\mathrm{T}}=\left\{\frac{1}{2} m_{1} g \sin \left(\theta_{1}\right) f_{H_{1}}(u) \quad \frac{1}{2} m_{2} g \sin \left(\theta_{2}\right) f_{H_{2}}(u)\right\} \\
& f_{d r}^{\mathrm{T}}=\left\{\mu_{r_{1}} m_{1} g f_{H_{1}}(\dot{u}) \quad \mu_{r_{2}} m_{2} g f_{H_{2}}(\dot{u})\right\} \\
& f_{d r}^{\mathrm{T}}=\left\{\mu_{s_{1}} N_{1} f_{H_{1}}(\dot{u}) \quad \mu_{s_{2}} N_{2} f_{H_{2}}(\dot{u})\right\} \\
& f_{H}(x)=\left\{\begin{array}{ccc}
1 & \text { if } & x \geq d \\
\frac{1}{d} x & \text { if } & -d<x<d \\
-1 & \text { if } & x \leq d
\end{array}\right.
\end{aligned}
$$

where $m$ is the mass supported by the isolator, $g$ is the gravitational acceleration, $\mu_{r}$ is the rolling friction coefficient, $\mu_{s}$ is the sliding friction coefficient, $N$ is the normal force applied on the sliding interface, $\theta$ is the angle of the inclined V-shape surfaces and $f_{H}$ is a function governed by a parameter called yield displacement $d$ and a auxiliar variable $x$ associated with the displacement and speed of the base of system. Additionally, the subscripts 1 and 2 denote the proper direction of each parameter in the horizontal plane.

In order to solve Eq.1, the numerical solver of ordinary differential equations (ODE's) ode23t implemented by MATLAB was used; it showed greater efficiency and numerical stability compared to other methods offered by MATLAB ODE-pack. In general, the algorithm of this solver is an implementation of the trapezoidal rule with an adaptive step-size associated with the use of a "free" interpolant (Shampine et al., 1999). The use of this solver requires that Eq. 1, which is a second order equation, be rewritten as a two first-order ODE system, as shown in the following expression:

$$
\dot{v}=A_{1} v(t)+A_{2} H(u, \dot{u})+A_{3} \ddot{u}_{g}
$$

with

$$
A_{1}=\left[\begin{array}{cc}
0 & I \\
-M^{-1} K & -M^{-1} C
\end{array}\right] ; A_{2}=\left[\begin{array}{c}
0 \\
-M^{-1} R
\end{array}\right] ; A_{3}=\left[\begin{array}{c}
0 \\
-\Gamma
\end{array}\right]
$$


where, $A_{1}$ and $\dot{v}(t)^{\mathrm{T}}\left(=\left\{\begin{array}{ll}u & \dot{u}\end{array}\right\}\right)$ represent the matrix of properties and response vector of the system in state-state representation. The non-linearity of the system is represented by $H(u, \dot{u})$ function, which includes the effects of the RB system.

\section{Validation of the motion equations}

To validate the numerical simulation algorithm, the results of an experimental evaluation Ortiz-Cano et al. (2015) and a numerical simulation of a building with and without RB isolation were compared. The characteristics of the building model and the numerical simulations are described next.

\subsection{Building description}

The physical model consists of a four-story-one-bay frame structure supported by polymeric columns with rectangular section of $20.0 \times 2.9 \mathrm{~mm}$. The model has a total height of $803.4 \mathrm{~mm}$ and floor area of $150 \times 200 \mathrm{~mm}$. The slabs and the story-column joins are constructed of aluminum plates. The base of the building has a thickness of $8.0 \mathrm{~mm}$, storys and story-column connections a thickness of $4.0 \mathrm{~mm}$. Also, the building lumped additional masses of $0.50 \mathrm{~kg}$ at second and third storys. A detailed outline of the geometry of the experimental model is showed in Figure 1.

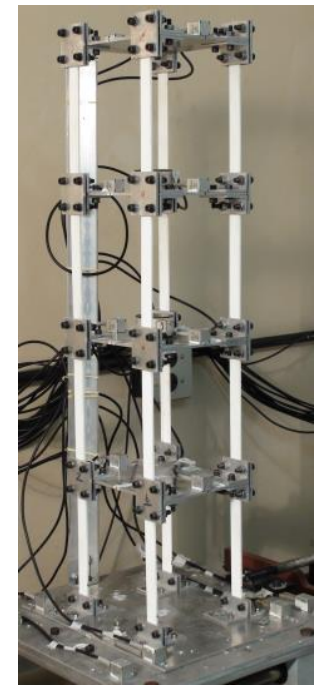

(a) Picture.

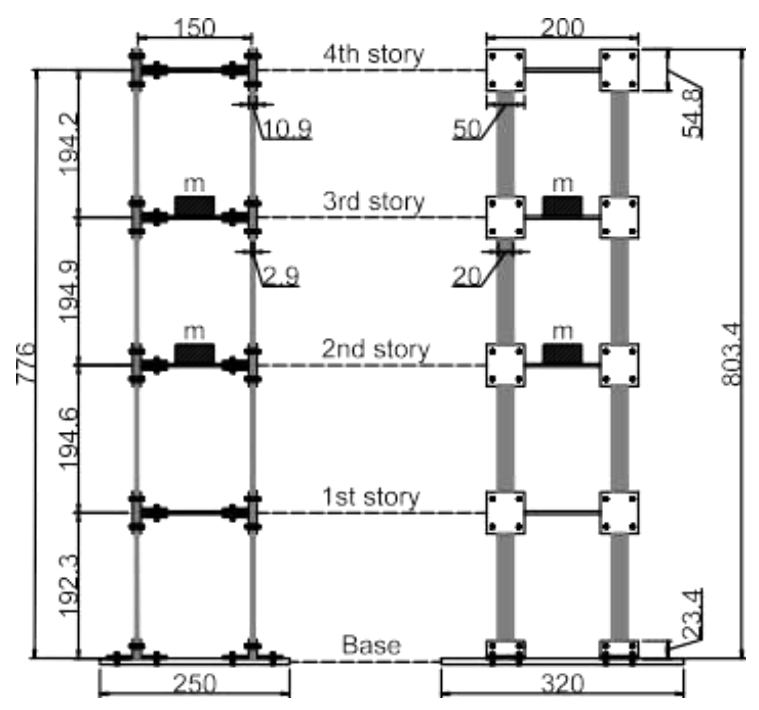

(b) Geometry, measures in $\mathrm{mm}$.

Fig. 1: Physical model. 
According to the experimental tests carried out and the analysis methodologies adopted by the author, the modal parameters identified for the fixedbase building model are summarized in Table 1.

Table 1: Identified dynamic properties of the building.

\begin{tabular}{ccc} 
Mode (Type-Direction) & $f_{\text {exp }}(\mathrm{Hz})$ & $\xi_{\text {exp }}(\mathrm{Hz})$ \\
\hline $1^{\text {st }}$ (Flexural - Weak) & $6.82 \pm 0.02$ & $0.59 \pm 0.03$ \\
$2^{\text {nd }}$ (Flexural - Weak) & $20.44 \pm 0.03$ & $0.51 \pm 0.01$ \\
$3^{\text {rd }}$ (Torsional) & $21.55 \pm 0.02$ & $2.88 \pm 0.03$ \\
$4^{\text {th }}$ (Flexural - Weak) & $31.70 \pm 0.02$ & $0.30 \pm 0.01$ \\
\hline
\end{tabular}

In addition, Rayleigh damping constants values of $\alpha=4.03 \times 10^{-1}$ and $\beta=$ $5.58 \times 10^{-5}$ were determined for the first two modes of vibration (which add up to $95 \%$ modal participation of the total mass of the building) because Rayleigh damping model is used to assembly the damping matrix $C$ within the numerical simulation algorithm.

\subsection{RB system description}

The seismic behavior of a bidirectional RB isolation system composed mainly of two surfaces and an array of rollers rolling in an orthogonal arrangement is investigated. Previous works have shown that the use of these isolation system enhances the dynamic response of the structure subjected to seismic excitations, e.g., Ortiz-Cano et al. (2014) and Menga et al. (2017). However, there are certain drawbacks to this isolation scheme, such as the self-centering mechanism and rolling bearing support stability (Sanchez-Torres et al., 2019).

Thereby, the bidirectional isolation system includes an intermediate bearing plate with a V-shaped tilted in both and a magnetic energy dissipation complement (Fig.2) that are proposed to attenuate the isolator displacement while simultaneously dissipating the energy induced by seismic loads. To avoid the pounding between the rollers and the $\mathrm{V}$-shaped intersection, an arc rolling zone is used with a fixed curvature radius higher than the roller radius being limited to keep a predominant linear sloping surface (Wang et al., 2014). 


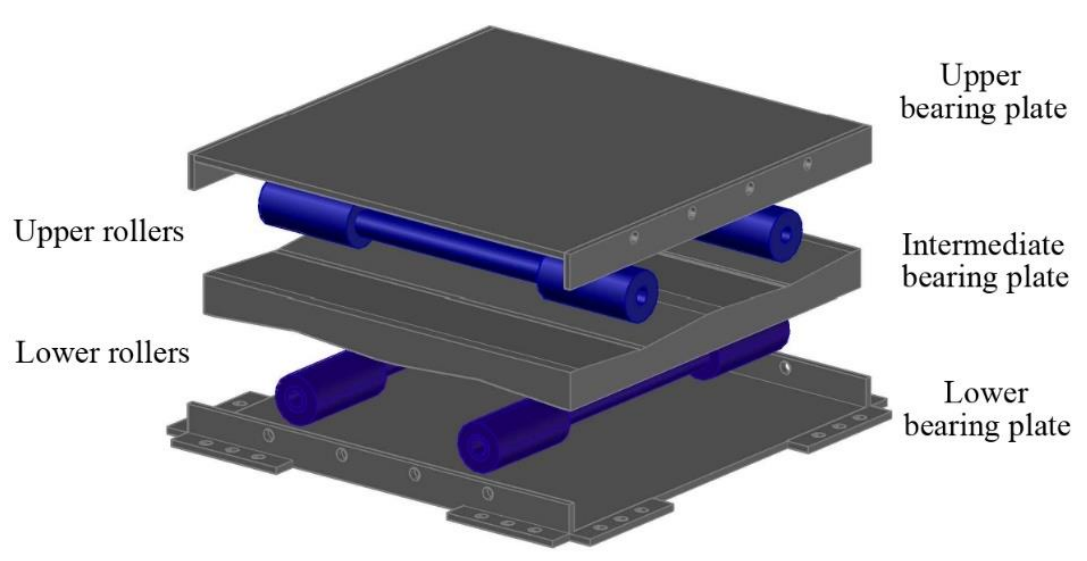

Fig. 2: Bidirectional RIS model with magnetic energy dissipation.

\subsection{Numerical modelling}

To numerically model the physical building model, 3D frame elements were considered, which are characterized by the elasticity modulus $E$, the Poisson ratio $v$ that defines the shear modulus $G$, the density $\rho$, the area $A$, the polar moment of inertia $J_{x}$ and the moments of inertia $I_{y}$ and $I_{z}$. The axis of the 3D frame elements in local coordinates coincides with the $x$ direction in global coordinates according to the adopted coordinate system shown implicitly in Figure 3. In the numerical representation of the physical model, three types of 3D frame elements were used, Table 2 lists characteristics of these.

Table 2: Mechanical and geometric characteristics of the elements.

\begin{tabular}{cccccccc}
\hline $\begin{array}{c}\text { Element } \\
\text { type }\end{array}$ & $E$ & $v$ & $\rho$ & $A$ & $J_{x}$ & $I_{y}$ & $I_{z}$ \\
& $(G P a)$ & $(-)$ & $\left(\mathrm{kg} / \mathrm{m}^{3}\right)$ & $\left(10^{-5} \mathrm{~m}^{2}\right)$ & $\left(10^{-10} \mathrm{~m}^{4}\right)$ & $\left(10^{-10} \mathrm{~m}^{4}\right)$ & $\left(10^{-10} \mathrm{~m}^{4}\right)$ \\
\hline 1 & 28 & 0.33 & 1800 & 5.9 & 1.5 & 20 & 0.4 \\
2 & 70 & 0.33 & 2700 & 40.0 & 77 & 1300 & 25 \\
3 & 70 & 0.33 & 0 & 40.0 & 77 & 1300 & 25 \\
\hline
\end{tabular}

Element's type 1 represents the columns constructed in polymeric material, while the element types 2 and 3 represent the aluminum connections and slabs defined as beams in the numerical model. The previous assignment of element types is presented schematically in Figure 3. It is important to highlight that the density of the elements type 3 takes the value of 0 because the slab masses, and other elements that provide mass on each story, were modelled as masses concentrated on the nodes of each story. Thus, concentrated masses of $539.3 \mathrm{~g}, 196.6 \mathrm{~g}, 321.2 \mathrm{~g}$, 
$321.1 \mathrm{~g}$ and $196.6 \mathrm{~g}$ were incorporated into each of the nodes of the base, $1^{\text {st }}, 2^{\text {nd }}$, $3^{\text {rd }}$ and $4^{\text {rd }} 4$ th story.

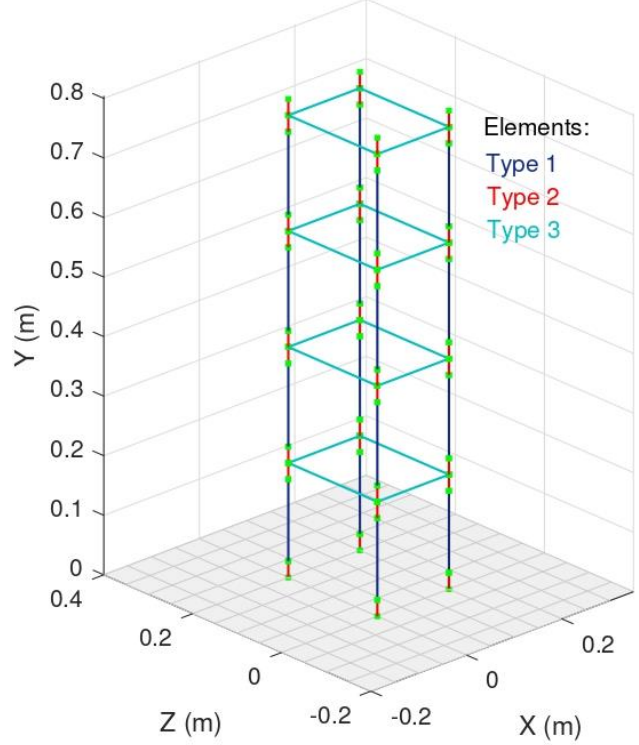

Fig. 3: Numerical model: 3D frame element types assigned.

\subsection{Model validations}

A comparative analysis of the numerical and experimental responses of the building, the RB system and the building with RB system is next presented.

\subsubsection{Building}

The validation of the numerical model corresponding to the fixed-base building was performed on frequencies and time domains; in addition, the frequency response functions (FRFs) and the acceleration response of the model stories were used. 


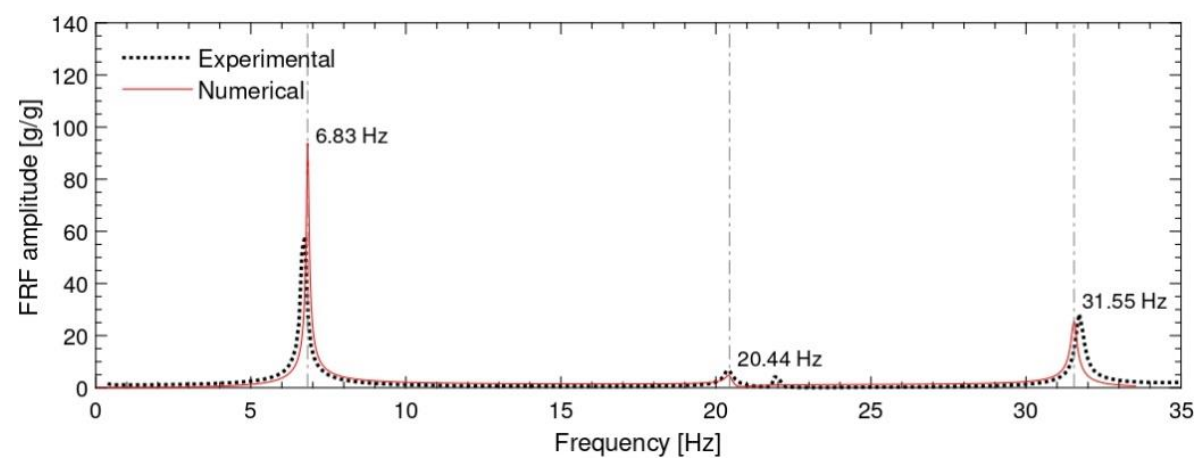

(a) with base excitation

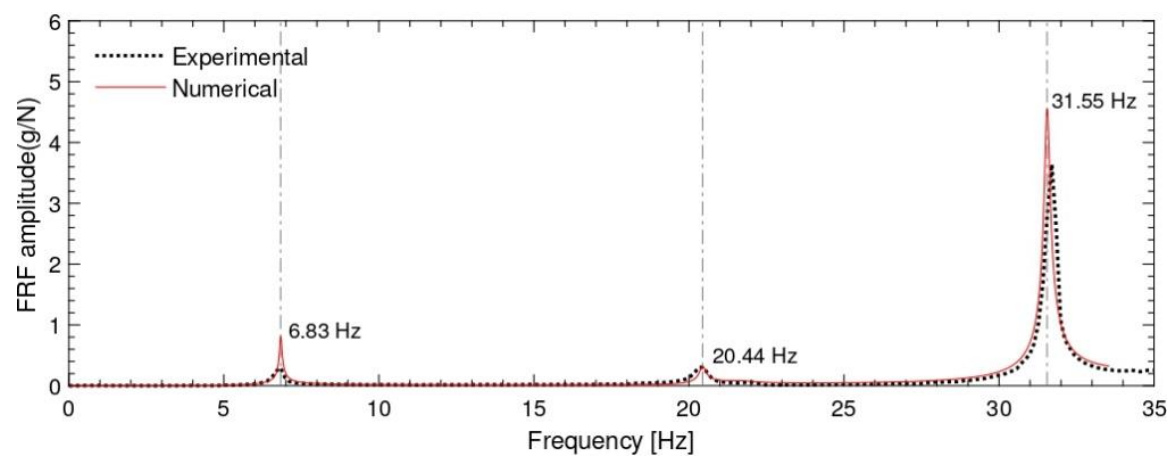

(b) with impact excitation in the $1^{\text {st }}$ story.

Fig. 4: FRFs comparison of the $3^{\text {rd }}$ story.

Figure 4 shows that the natural vibration frequencies obtained numerically correspond to experimental measures with a maximum difference of $0.4 \%$. Regarding FRFs peaks values, the highest and lowest difference was of $60 \%$ and $11 \%$ for 1 st and 3rd modes in Figure $4 \mathrm{a}$ and of $200 \%$ and $25 \%$ for $1^{\text {st }}$ and $2^{\text {nd }}$ modes in Figure 4b. In general, the energy (FRF amplitude) follows the captured behavior of experimental results for all modes and natural vibration frequencies of the numerical model. Therefore, the numerical model is successfully validated in frequency domain terms for fixed base conditions.

The acceleration obtained by the proposed numerical model and experimental measures under white noise base excitation are presented in Figure 5. It is shown that the acceleration estimated for the numerical simulation are in good agreement with the experimental response in time despite of the acceleration amplitude reached. Thus, the numerical model is adequately validated in time domain. 


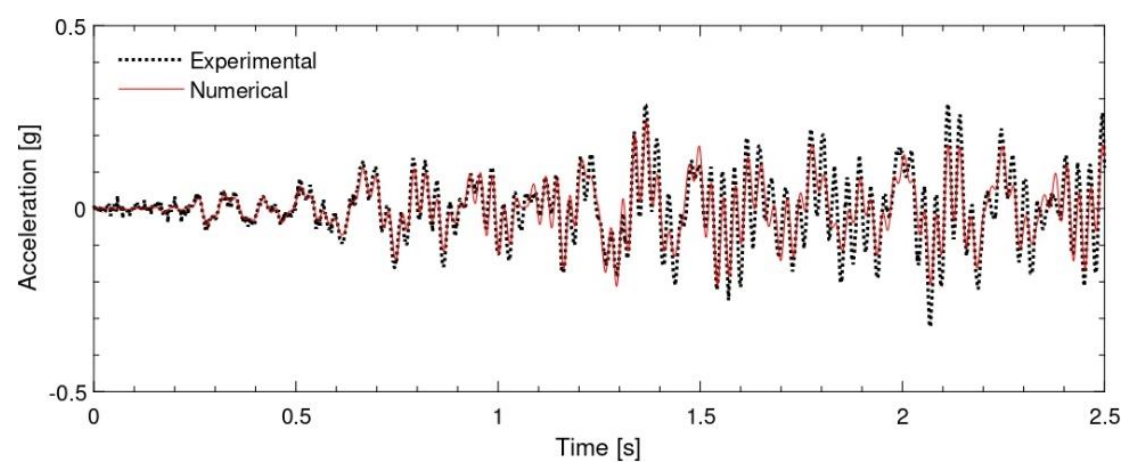

Fig. 5: Time series of acceleration response of the $3^{\text {rd }}$ story under base excitation.

\subsubsection{RB system}

The damping provided by the friction between the roller and the sloping surfaces is estimated from the experimental acceleration response of the RB system on free vibration. An initial displacement is applied at once for both horizontal directions. The rolling friction parameter $\mu_{r}$ in the numerical model was tuning using an iterative manual scheme with the aim of match the RB response in both directions. A value of $\mu_{r}=0.0057$ was found to be suit- able for each motion direction. To validate the dynamical behavior of the bidirectional RB isolation system, masses of $1.90 \mathrm{~kg}$ and $2.78 \mathrm{~kg}$ were considered on the intermediate plate and lower plate, respectively. These values correspond to the physical properties of the built system.

The free vibration response of the physical and numerical model is compared in Figure 6. It is observed that in both directions the numerical representation follows the pattern recorded in experimental test at the start. Also, the maximum acceleration in both direction is well capture in the numerical model. However, a gap is observed between numerical simulation and experimental response of RB system as a consequence of imperfections in rollers bearings and V-shape surfaces. In addition, some spikes appeared in the recorded acceleration due to the bidirectional effect of two components of movements (Wang et al., 2014). 


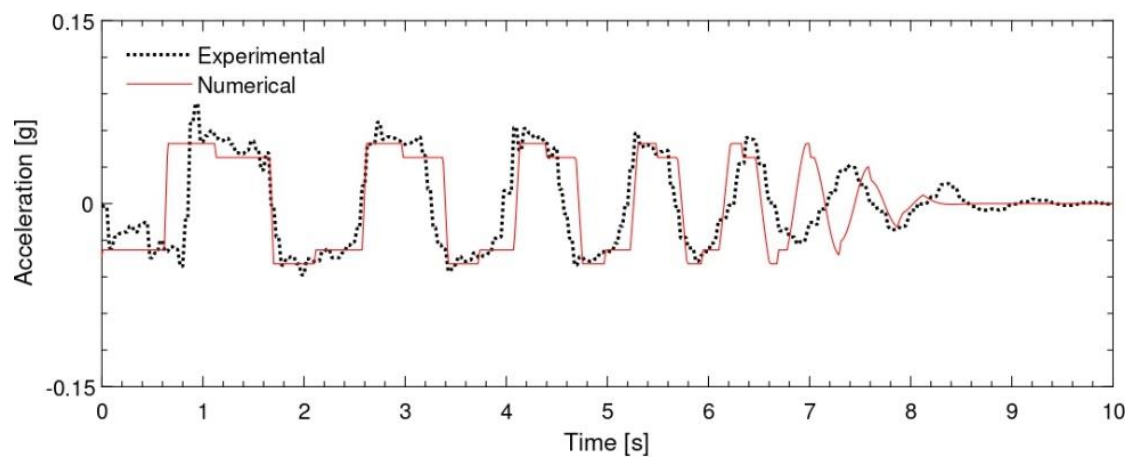

(a) response in $x$ direction

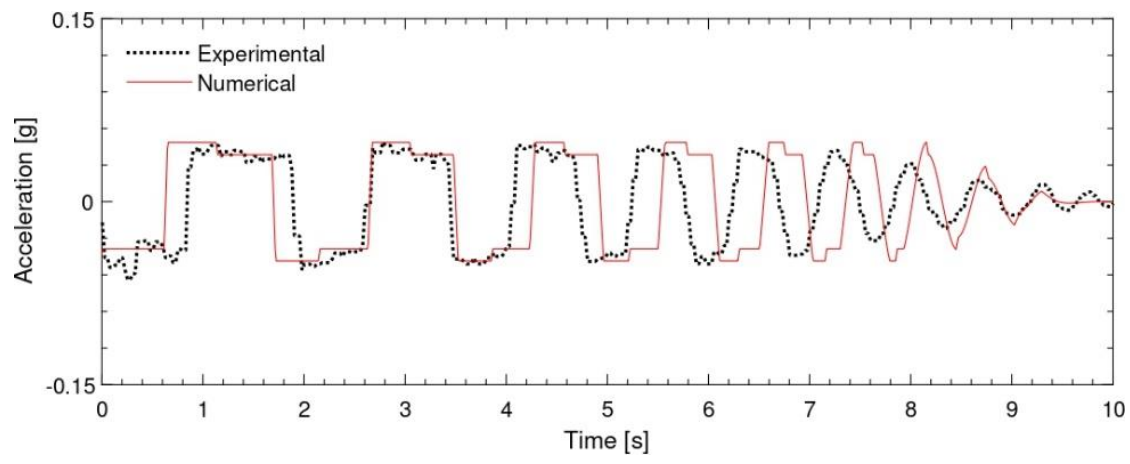

(b) response in $y$ direction

Fig. 6: Time series of acceleration response of the RB in free vibration.

The period value of several developed RB devices is constant for each particular geometry and prototype, e.g., period value of $2.01 \mathrm{~s}$ for a concave bearing surface evaluated by Hosseini and Soroor $(2011,2013)$. By contrast, the linear sloped RB device does not have a particular frequency as an important advantage.

Figure 6 shows that a time lag arises between numerical and experimental acceleration when the acceleration of RB system evolves in time. Figure $6 a$ is shown after $6.50 s$ that the repetitive path of numerically simulated response advanced the experimental acceleration record, i.e., the damping of RB device reduces with time. An opposite pattern is observed in the orthogonal direction, i.e., the response in $y$ direction (Fig.6a) after $4.00 \mathrm{~s}$ shows that the square form of numerical acceleration has a time delay with the experimental acceleration, i.e., the damping of RB device increases as the displacement of $\mathrm{RB}$ is reducing.

In this way, large sources of friction mechanism in RB systems have not been isolated; consequently, most of them are still roughly incorporated in a model (Zhang and Ali, 2021). Moreover, during the test of RB system, it was observed that as the rollers reduce its displacement and it is within arc rolling zone, the oscillatory 
movement of rolling bearing system trend to be unbalanced and compromise the accuracy of numerical modelling.

\subsubsection{Building with RB system}

The acceleration response of building coupled with RB system is compared in free base vibration (Figure 7a) and subjected to a white noise base excitation (Figure $7 \mathrm{~b}$ ). It is seen that the simulated responses follow the trend of the target values for kind of Dynamic load condition, which are particularly important for the performance of the simulated response used in the analysis.

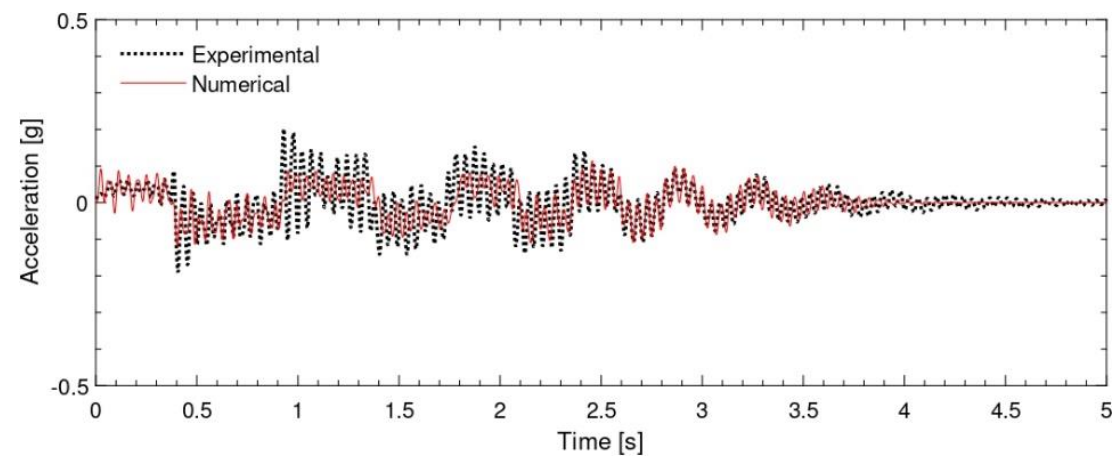

(a) with $\mathrm{RB}$ in free vibration.

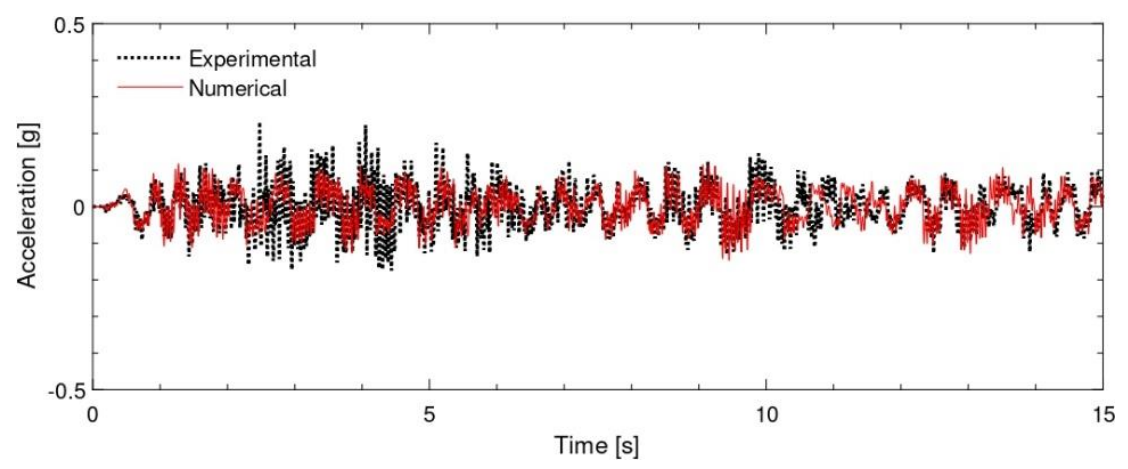

(b) with RB under base excitation.

Fig. 7: Time series of acceleration response of the $2^{\text {nd }}$ story.

Additional details about the numerical model validation for unidirectional or bidirectional base excitations components are presented by Ortiz-Cano et al. (2014), Ortiz-Cano et al. (2015), Sanchez-Torres et al. (2019) and González-Olaya et al. (2020). 


\section{RB system performance under near-fault earthquakes}

In this section, the performance of the RB system in reducing the structural response of buildings subjected to a particular seismic excitation is evaluated through an extensive numerical study. In this study, two isolated structures with different configurations of the RB system were simulated when subjected to the horizontal components of two near fault seismic records.

The two structures considered are the product of the partial modification of the validated numerical model to obtain: (I) an S1 structure in which the vibration frequencies of its first two modes are less than $2.0 \mathrm{~Hz}$; and (II) an S2 structure in which the vibration frequencies of its first two modes are greater than $10.0 \mathrm{~Hz}$, representing buildings with low and high lateral stiffness, respectively. The modification was made by affecting the inertias $I_{y}$ and $I_{z}$ of the elements type 1 . For the S1 structure $I_{y}$ was reduced to $3.0 \times 10^{-12} \mathrm{~m}^{4}$ and $I_{z}$ to $2.0 \times 10^{-12} \mathrm{~m}^{4}$, while for the S2 structure $I_{y}$ was reduced to $1.0 \times 10^{-10} \mathrm{~m}^{4}$ and $I_{z}$ was increased to $1.0 \times$ $10^{-10} \mathrm{~m}^{4}$. Thus, Table 3 shows the predominant frequencies of the modified structures that correspond to the first three modes of vibration.

Table 3: Modes and frequencies of vibration of structures S1 and S2.

\begin{tabular}{ccc}
\hline & \multicolumn{2}{c}{ Frequency $(\mathrm{Hz})$} \\
Mode (Type-Direction) & $\mathrm{S} 1$ & $\mathrm{~S} 2$ \\
\hline $1^{\text {st }}$ (Flexural - Weak) & 1.51 & 10.21 \\
$2^{\text {nd }}$ (Flexural - Weak) & 1.85 & 10.30 \\
$3^{\text {rd }}$ (Torsional) & 3.09 & 10.73 \\
\hline
\end{tabular}

Near fault seismic events are generally characterized by exhibiting a longduration, large-magnitude displacement pulse with large accelerations and limited frequencies content compared to far fault earthquakes. Given these characteristics of ground displacement and acceleration, large lateral displacements are expected in base seismic isolation systems when they are subjected to this type of excitation, which results in an inefficient system. Therefore, it can be stated that the most critical operating condition for base isolation systems is when these seismic events occur.

Considering the above, the present numerical study uses near fault earthquake horizontal records to evaluate the performance of RB in the most unfavorable base excitation conditions. Table 4 presents the selected near fault seismic records obtained from PEER Ground Motion Database (Ancheta et al., 2013). Vertical ground motions components are not used because it has a negligible effect 
performance of different isolation devices (Ou et al., 2010; Beirami-Shahabi et al., 2019).

Table 4: Near fault earthquake records.

\begin{tabular}{|c|c|c|c|c|}
\hline Data & \multicolumn{4}{|c|}{ Earthquake } \\
\hline Event name & \multicolumn{2}{|c|}{ Northridge - 01} & \multicolumn{2}{|c|}{ Imperial Valley - 06} \\
\hline Date & \multicolumn{2}{|c|}{ January 17, 1994} & \multicolumn{2}{|c|}{ October 15, 1979} \\
\hline Record & \multicolumn{2}{|c|}{ Newhall - Fire Station } & \multicolumn{2}{|c|}{ El Centro - Array \#5 } \\
\hline Component & 90 & 360 & 140 & 230 \\
\hline PGD $(\mathrm{cm})$ & 17.6 & 34.3 & 48.9 & 75.2 \\
\hline PGA $(g)$ & 0.566 & 0.590 & 0.529 & 0.383 \\
\hline
\end{tabular}

Since S1 and S2 are defined from a physical model, the selected seismic records are scaled in displacement amplitude to $5 \%$ of their PGD to conserve the magnitudes. Real records were scaled using the Make Quake program from Quanser Consulting Inc. (2010) that implements the scaling algorithm developed by Kausel and Ushijima (1979) and that allows to preserve both the accelerations magnitude and frequencies content of the original records.

First, the S1 and S2 structures were simulated subjected to scale excitations under fixed base conditions. Then, they were simulated incorporating the RB systems considering four angles of inclination of the V-shaped surfaces: $1^{\circ}, 2^{\circ}, 3^{\circ}$ and $4^{\circ}$. Thus, in Figures 8 and 9 the acceleration response of th $4^{\text {th }} e$ story and the hysteresis diagram of the RB system are presented for each structure and base excitation.

In these figures it is observed that all the configurations manage to reduce the structural response of S1 and S2. System efficiency increases as the angle of inclined surfaces decreases, achieving a reduction in peak acceleration from $80 \%$ to $95 \%$ with the lowest angle of inclination. For S2 the reduction is appreciably greater when compared to $\mathrm{S} 1$ for all the evaluated inclinations. Additionally, for S2 the increase in response reduction varies slightly with the angle of inclination. In terms of base displacements, it is observed that these also decrease as the inclination of the inclined surfaces decreases, however, these displacements are excessive since they are equal to or greater than scaled peak ground displacement (PGD), Figures 8c, 8d, 9c and 9d. 


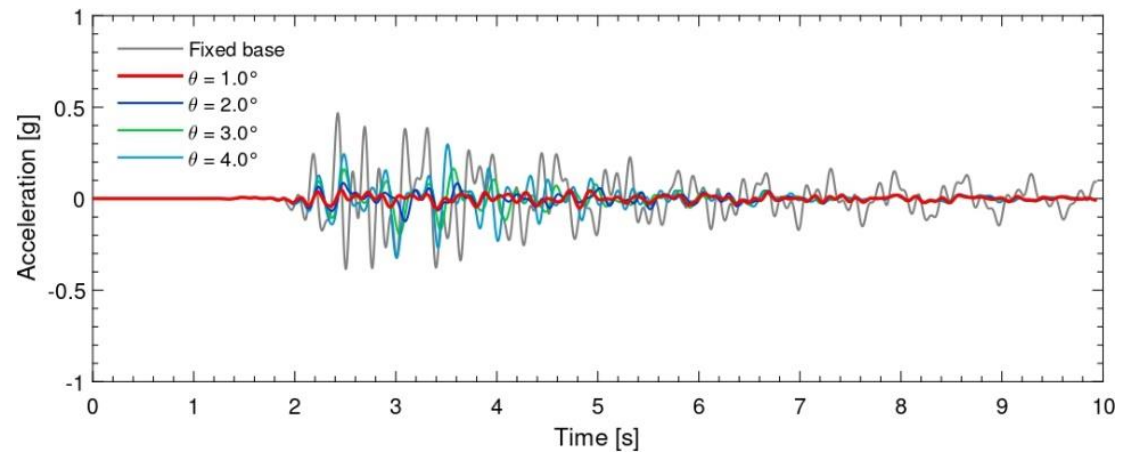

(a) Total acceleration response of $4^{\text {th }}$ story in strong direction.

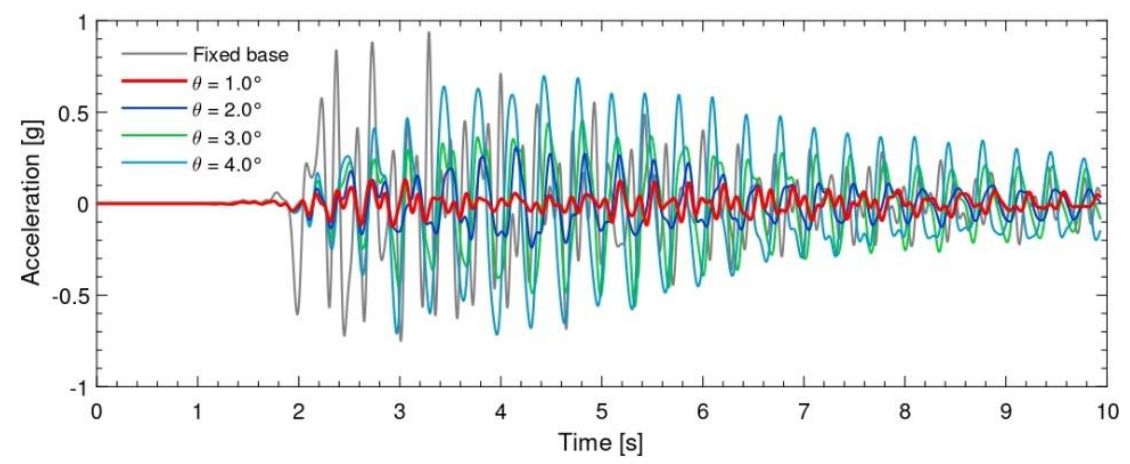

(b) Total acceleration response of $4^{\text {th }}$ story in weak direction.

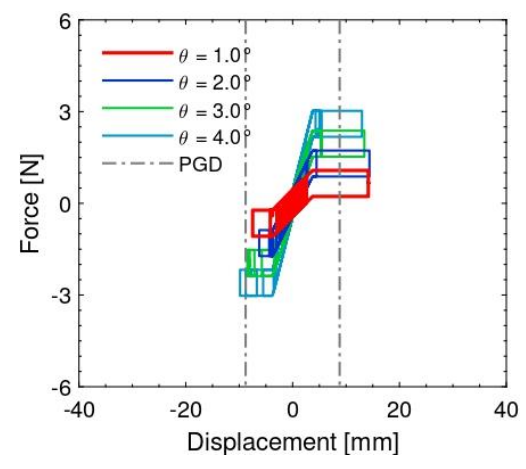

(c) RB hysteresis diagram in strong direction

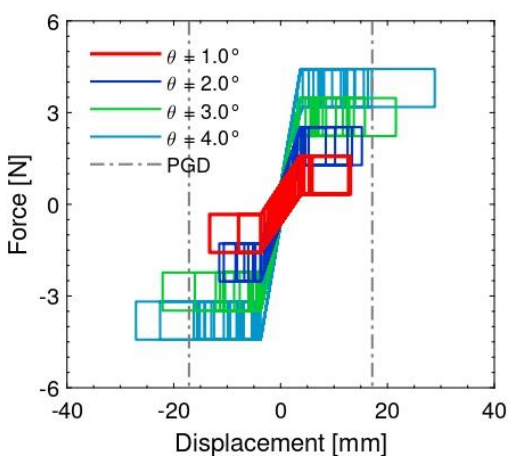

(d) RB hysteresis diagram in weak direction

Fig. 8: Response of structure S1 subjected to the scaled earthquake record Northridge (1994) Newhall Fire Station. 


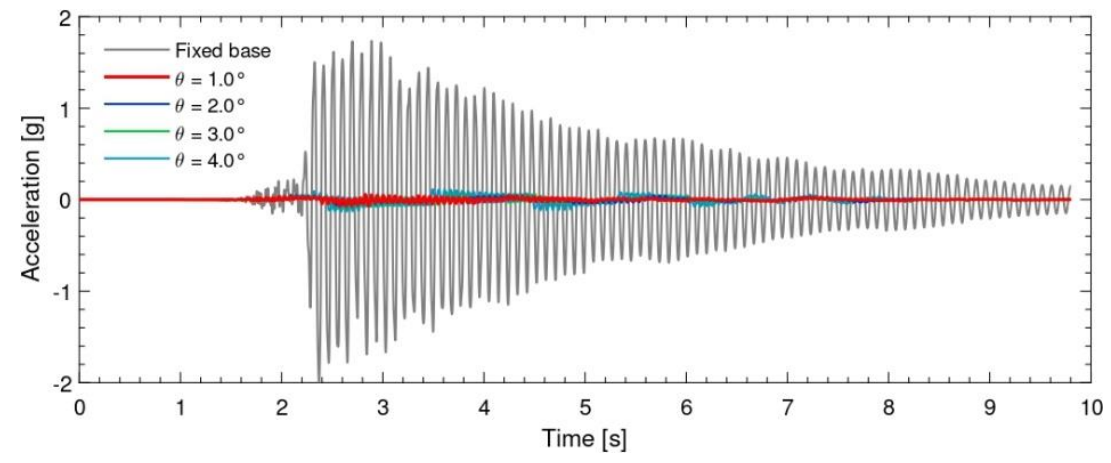

(a) Total acceleration response of $4^{\text {th }}$ story in strong direction.

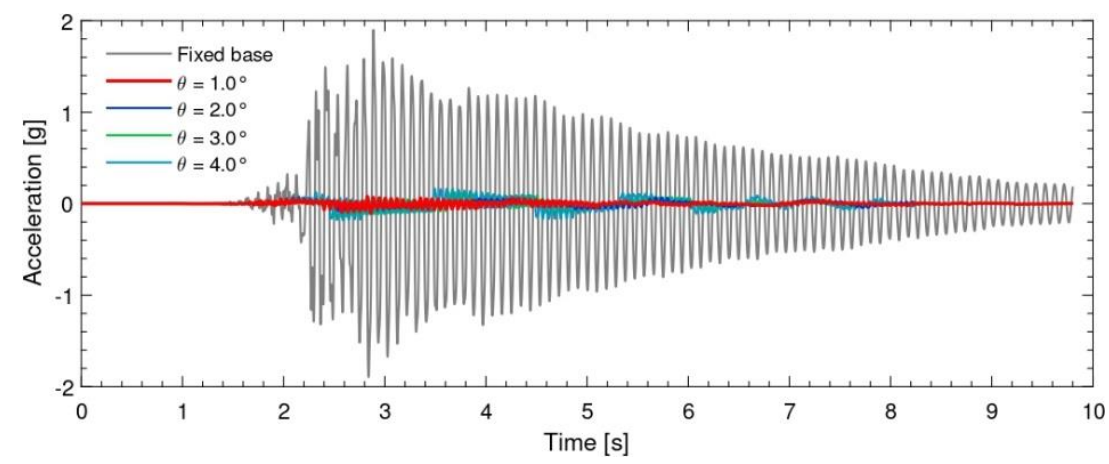

(b) Total acceleration response of $4^{\text {th }}$ story in weak direction.

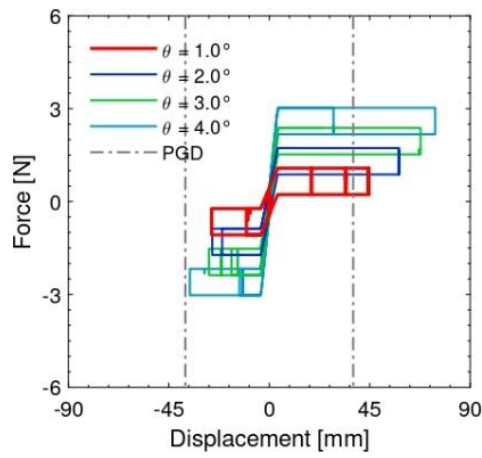

(c) RB hysteresis diagram in strong direction

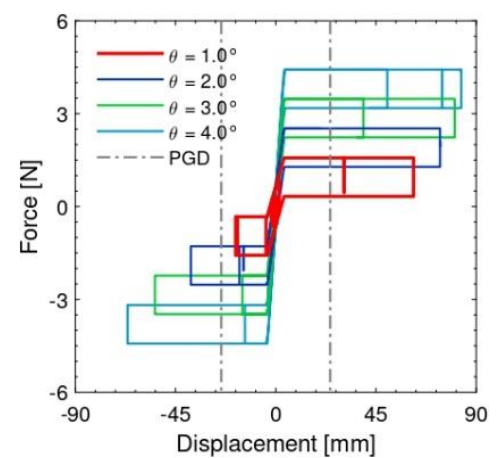

(d) RB hysteresis diagram in weak direction

Fig. 9: Response of structure S2 subjected to the scaled earthquake record Imperial Valley (1979) El Centro Array \#5.

On the other hand, in Figure $8 \mathrm{~b}$ it is evident that even when the peak acceleration is reduced by the RBs, the amplitude of the response of the isolated structure over the excitation time increases for the inclination angles greater than and equal to $3.0^{\circ}$. Given the above, the root-mean-square (RMS) is chosen as an appropriate measure that allows evaluating the entire response and not just peak values. From this point on, the performance of the RB system is analyzed in terms 
of the RMS for each of the simulations carried out. The effectiveness in controlling the structural response with the RB system is quantified through the following expression:

$$
C E[\%]=\frac{\sigma_{u}-\sigma_{c}}{\sigma_{u}}
$$

Where $\sigma_{c}$ and $\sigma_{u}$ are the RMS values of the structural response with and without the RB system, respectively. Furthermore, $\sigma_{c}$ and $\sigma_{u}$ for each story of the structure is calculated as the vector sum of RMS value in each horizontal directions of motion, i.e., $\sqrt{\sigma_{x}^{2}+\sigma_{z}^{2}}$.

Processing each acceleration responses obtained from simulations carried out through Equation 10, Figures 10 and 11 present the efficiency in the control of the structural response of the RBs for different angles of the inclined surfaces on each of the stories.

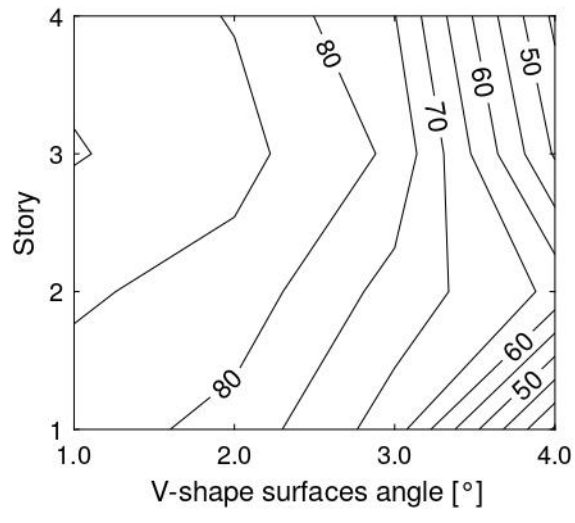

(a) Imperial Valley (1979) El Centro Array\#5.

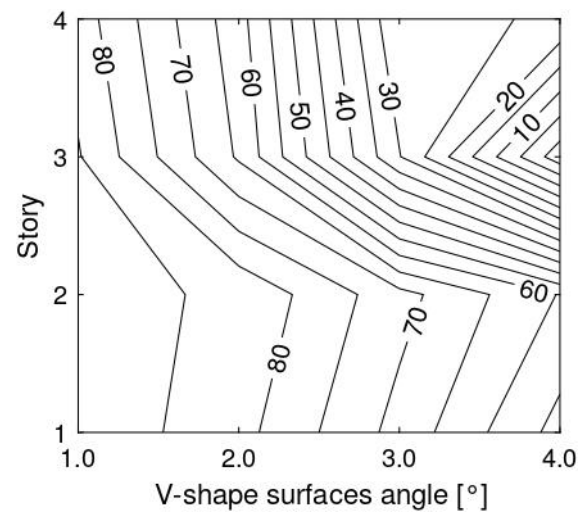

(b) Northridge (1994) Newhall Fire Station.

Fig. 10: Contour plot of acceleration control effectiveness versus V-shape surfaces angle for S1 structure. 


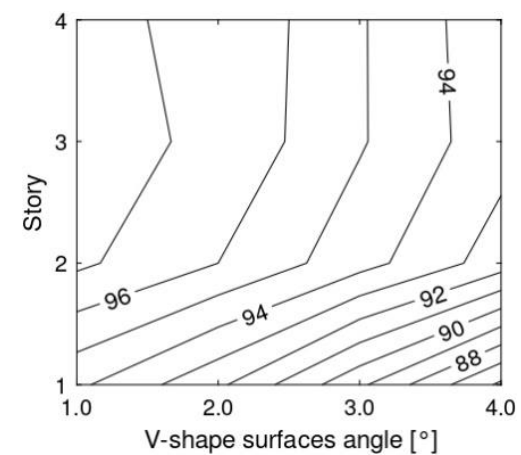

(a) Imperial Valley (1979) El Centro Array\#5.

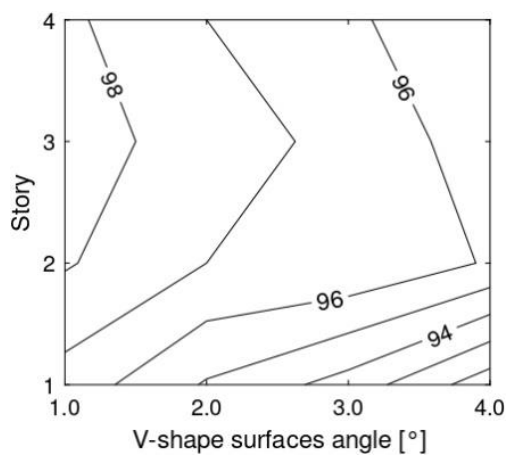

(b) Northridge (1994) Newhall Fire Station.

Fig. 11: Contour plot of acceleration control effectiveness versus V-shape surfaces angle for $\mathrm{S} 2$ structure.

In the S1 structure, the efficiency of the RBs with the evaluated angles can reach reductions from 5\% to $85 \%$ (Fig.10). Again, the efficiency increases as the angle of inclination of the inclined surfaces is smaller. RB efficiency drops dramatically for angles from $3.0^{\circ}$ to $4.0^{\circ}$. From an angle of $4.0^{\circ}$, an amplification of the response can be generated following the trend of the contour plot when considering the scaled excitation Northridge (1994) Newhall Fire Station (Fig. 10b). On the other hand, a prior study reported that a larger angle of inclination did not diminish peak displacement without supplementary energy dissipation devices added and additional damping in the isolation devices needs to be investigated to adjust the isolation system's performance for different levels of friction forces higher than the provided by the friction of the rollers (Ou et al., 2010; Harvey-Jr and Gavin, 2015).

For the S2 structure, the RB system can achieve reductions close to $99 \%$ with an inclination angle of $1.0^{\circ}$ (Fig. 11b). If the angle of inclination of the surfaces increases, its efficiency slightly decreases, achieving at least a reduction of $86 \%$ for the critical story when considering an angle of inclination of $4.0^{\circ}$ and the scaled excitation Imperial Valley (1979) El Centro Array \# 5 (Fig. 11a).

On the other hand, as large displacements in the base were evidenced for the two structures studied, a set of simulations of the structural response was carried out by including in the RBs an energy dissipation system from friction surfaces to control base displacements. It was found that for the S1 structure any combination of the angle of inclination of the inclined surfaces with an energy dissipation force that appreciably reduced the base displacement would lead to an amplification of its response. 
In the S2 structure, a significant reduction in structural response together with an appreciable reduction in base displacements can be achieved by combining inclination angles greater than 4.0 . If smaller angles are used, self- centering capacity of the system would be compromised, and non-recoverable base displacements would be generated at the end of the excitation. In general, the restoring force produced by the inclination of the V-shaped surfaces should not be exceeded by the dissipation force developed in the friction plates since the recentering force at the end of the excitation would be insufficient to overcome the force of friction of the dissipation system.

In Figure 12, the efficiency of an RB system with an angle of inclination of the $V$-shaped surfaces of $4.0^{\circ}$ is presented against different forces of the friction plate system for the structure control of the S2 structure response. In this figure, the force developed by the energy dissipation system is normalized with the mass supported by the RBs in each direction.
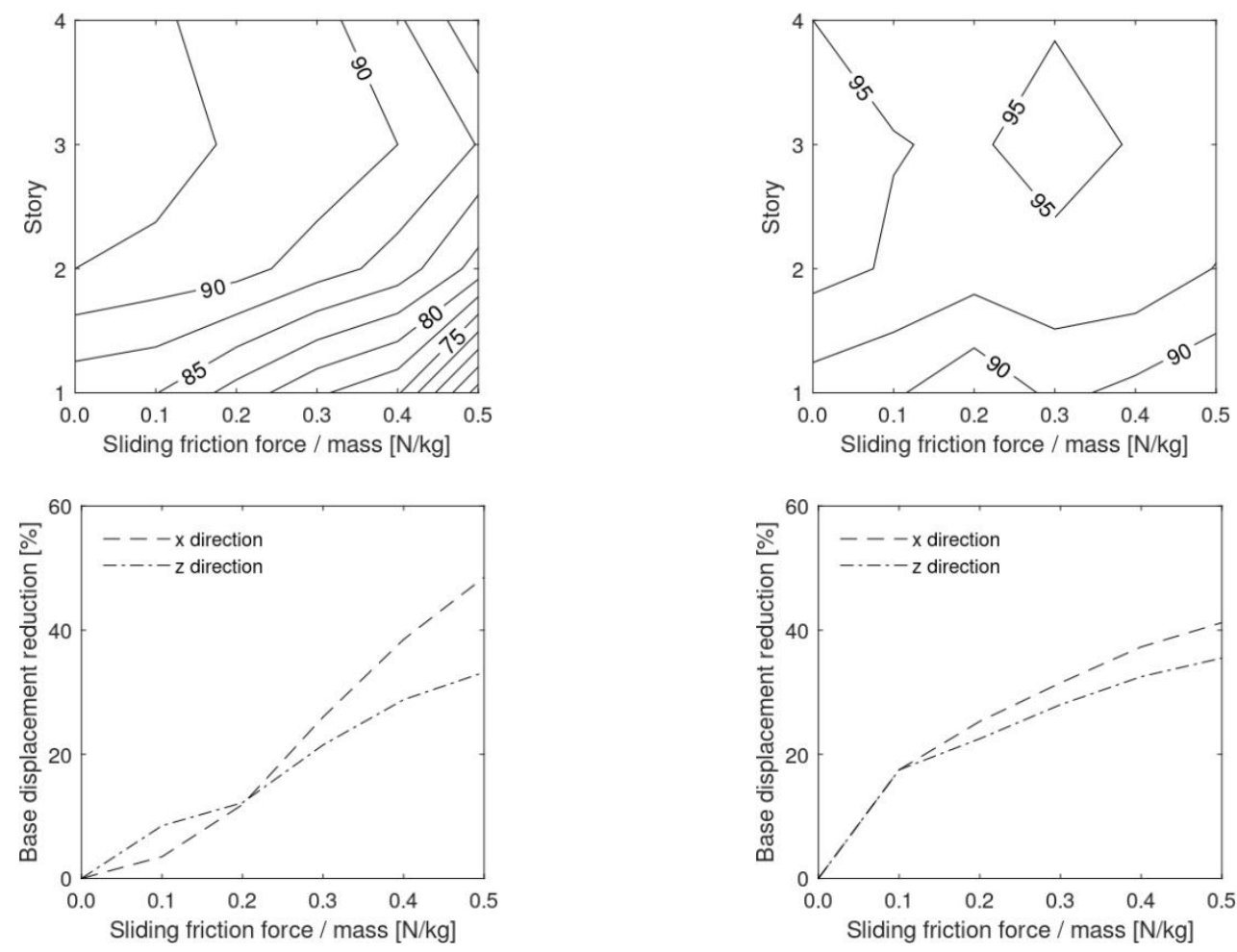

(a) Imperial Valley (1979) El Centro Array\#5.

(b) Northridge (1994) Newhall Fire Station.

Fig. 12: Contour plot of acceleration control effectiveness versus sliding friction per supported mass with $\theta=4.0^{\circ}$ for S2 structure.

It is observed that the efficiency in the control of the response in accelerations decreases and the control of the base displacements increases as the force developed in the friction dissipation system is greater; additionally, the control of the 
base displacements increases more slowly as sliding friction force is greater. Figures $12 \mathrm{a}$ and $12 \mathrm{a}$ are shown that the maximum displacement reached by the sloped rolling rod base isolator is related to the peak ground accelerations of the earthquake motion, i.e., the lowest performance is achieved when the structure is subject to Imperial Valley (1979) El Centro Array \#5 scale record, when it is designed with a larger sloping angle and a higher sliding friction force/mass as has been recently reported, e.g., Wang et al. (2019).

Given the above, a suitable combination that achieves the greatest reduction in the response of the structure with the minimum base displacement can be achieved by varying the inclination of the $V$-shaped surfaces and the energy dissipation force. For the case of the analyzed structure, with the maximum control of the base displacement, the response in accelerations of the structure can be reduced in each of its floors from $60 \%$ to $87 \%$ in the most critical condition base excitation, i.e., Northridge (1994) Newhall Fire Station.

\section{Conclusions}

This paper presents an extensive numerical study to evaluate the performance of the RB system in reducing the structural response of multi-column systems subjected to the horizontal components of near-fault earthquake records. The reduction of the structural response was evaluated through direct comparison and the RMS statistical measure between the simulation results under fixed base conditions and using the RB system. Thus, the following conclusions are drawn from the present study:

1. The RB system manages to significantly reduce the response of the evaluated structures when subjected to near-fault earthquake excitations. Direct comparison shows reductions in peak acceleration of up to $80 \%$ for the flexible structure and $98 \%$ for the rigid structure. In terms of the RMS measurement, acceleration reductions of $5 \%$ to $85 \%$ are achieved for the flexible structure, while in the rigid structure reductions of $86 \%$ to $96 \%$ are achieved.

2. In the rigid structure the efficiency of the RB system is significantly higher than in the flexible structure, however, there are base displacements equal to or greater than the earthquake ground peak displacement in both cases.

3. One way to control the displacements generated at the base is the use of energy dissipation systems. In the numerical study, frictional energy dissipation systems were simulated; they were able to reduce approximately $35 \%$ of the base displacements and guarantee at least a $60 \%$ RMS reduction in the total acceleration of the rigid structure. For the flexible structure it was found that a reduction in the base displacements will inevitably lead to the amplification of the response with respect to the fixed base condition, which 
indicates that using this displacement control system is not suitable for this particular situation.

4. It is recommended to use friction systems for energy dissipation on surfaces that are sufficiently inclined to guarantee an appropriate self-centering capacity of the RB system. In the numerical study, it was found that angles less than or equal to $3.0^{\circ}$ generated residual displacements at the base due to loss of self-centering capacity for the rigid structure.

5. An adequate relationship between the angle of the inclined surfaces and the parameters associated with the energy dissipation system will result in a maximum reduction in the response of total acceleration with the minimum base displacement for a particular building and a set of seismic excitations.

Several aspects of the use of RB systems in multi-column structures were studied and presented; however, this work opens the door to future research, both numerical and experimental. Some future aspects that can be addressed are:

1. Experimentally validate the results obtained numerically for a reduced and a full scale.

2. Consider vertical accelerations effects induced by seismic excitation and by the behavior of the roller bearings.

3. Evaluate numerically and experimentally the use of RB in non-symmetric structural systems whose predominant vibration modes of vibration are torsional type.

4. Develop an energy dissipation system that reduce the base displacements with a minimum impact on the control efficiency of total acceleration of the buildings.

\section{Acknowledgments}

This research was supported by the Office Vice-Provost for Research at Universidad Militar Nueva Granada under grant number INV-ING-2982, this support is gratefully acknowledged.

\section{References}

Ancheta T, Darragh R, Stewart J, Seyhan E, Silva W, Chiou B, Wooddell K, Graves R, Kottke A, Boore D, Kishida T, Donahue J. (2013). PEER NGA-West2 Database. Tech. Rep. PEER 2013/03, Pacific Earthquake Engineering Research Center (PEER), PEER Core Institutions, Berkeley, CA, USA.

Andreaus U, De Angelis M. (2020). Influence of the characteristics of isolation and mitigation devices on the response of single-degree-of-freedom vibro-impact systems with two-sided bumpers and gaps via shaking table tests. Structural Control and Health Monitoring 27(5):e2517. 
Bagerzadeh-Karimi M, Geneş M. (2019). Probabilistic behavior assessment of baseisolated buildings and base isolation systems subjected to various earthquakes with different components. Arabian Journal for Science and Engineering 44(10):82658288.

Beirami-Shahabi A, Zamani-Ahari G, Barghian M. (2019). Suspended columns for seismic isolation in structures (SCSI): A preliminary analytical study. Earthquakes and Structures 16(6):743-755.

Beirami-Shahabi A, Zamani-Ahari G, Barghian M (2020) Base isolation sys- tems: A state of the art review according to their mechanism. Journal of Rehabilitation in Civil Engineering 8(2):37-61

Brewick P, Johnson E, Sato E, Sasaki T. (2020). Modeling the dynamic behavior of isolation devices in a hybrid base-isolation layer of a full-scale building. Journal of Engineering Mechanics 146(11):04020127.

Calhoun S. (2018). Evaluation of rolling-type isolation systems for seismic hazard mitigation. Master's thesis, The University of Oklahoma, Norman, OK, USA.

Calhoun S, Tehrani M, Harvey-Jr P. (2019). On the performance of double rolling isolation systems. Journal of Sound and Vibration 449:330-348.

Chen P, Hsu S, Zhong Y, Wang S. (2021). Real-time hybrid simulation of smart baseisolated raised floor systems for high-tech industry. Smart Structures and Systems 23(1):91-106

Erdik M, Ulker O, Şadan B, Tuzun C. (2018). Seismic isolation code developments and significant applications in Turkey. Soil Dynamics and Earthquake Engineering 115:413-437.

González-Olaya R, Ortiz-Cano N, Nieto-Leal A, Gaviria-Mendoza C. (2020). Numerical performance evaluation of a bi-directional roller seismic isolation bearings. In: 11th International Conference on Structural Dynamics, European Association for Structural Dynamics.

Harvey-Jr P, Gavin H. (2015). Assessment of a rolling isolation system using reduced order structural models. Engineering Structures 99:708-725.

Hosseini M, Soroor A. (2011). Using orthogonal pairs of rollers on concave beds (OPRCB) as a base isolation system Part I: Analytical, experimental and numerical studies of (OPRCB) isolators. The Structural Design of Tall and Special Buildings 20:928-950. 
Hosseini M, Soroor A. (2013). Using orthogonal pairs of rollers on concave beds (OPRCB) as a base isolation system Part II: Application to multi-story and tall buildings. The Structural Design of Tall and Special Buildings 22:192-216.

Kausel E, Ushijima R. (1979). Baseline correction of earthquake records in the frequency domain. Tech. Rep. R79-34, Massachusetts Institute of Technology, Cambridge, MA, USA.

Lee G, Ou Y, Niu T, Song J, Liang Z. (2010). Characterization of a roller seismic isolation bearing with supplemental energy dissipation for highway bridges. Journal of Structural Engineering 136(5):502-510.

Matsagar V, Jangid R. (2008). Base isolation for seismic retrofitting of structures. Practice periodical on structural design and construction 13(14):175- 185.

Menga N, Foti D, Carbone G. (2017). Viscoelastic frictional properties of rubber-layer roller bearings (RLRB) seismic isolators. Meccanica 52:2807-2817.

Naeim F, Kelly J (1999) Design of seismic isolated structures: From theory to practice. Wiley \& Sons, New York, USA.

Ortiz-Cano N, Magluta C, Roitman N. (2014). Numerical and experimental studies of a building with elastomeric and roller seismic isolation bearing. In: 9th International Conference on Structural Dynamics, European Association for Structural Dynamics.

Ortiz-Cano N, Magluta C, Roitman N. (2015). Numerical and experimental studies of a building with roller seismic isolation bearings. Structural Engineering \& Mechanics 54(3):475-489.

Ou Y, Song J, Lee G. (2010). A parametric study of seismic behavior of roller seismic isolation bearings for highway bridges. Earthquake Engineering \& Structural Dynamics 39(5):541-559.

Quanser Consulting Inc. (2010). Make Quake Program.

Rawat A, Matsagar V. (2021). An oblate spheroid base isolator and floating surface diaphragm for seismic protection of liquid storage tank. Journal of Earthquake Engineering pp 1-29.

Rawat A, Ummer N, Matsagar V. (2018). Performance of bi-directional elliptical rolling rods for base isolation of buildings under near-fault earthquakes. Advances in Structural Engineering 21(5):675-693.

Ryan K, Okazaki T, Coria C, Sato E, Sasaki T. (2018). Response of hybrid isolation system during a shake table experiment of a full-scale isolated building. Earthquake Engineering and Structural Dynamics 47:2214-2232. 
Sanchez-Torres D, Rico-Caviedes A, Ortiz-Cano N, Nieto-Leal A, Gaviria- Mendoza C. (2019). Assessment of a roller seismic isolation bearing for buildings under bidirectional excitations. In: XL Ibero-Latin-American Congress on Computational Methods in Engineering, Brazilian Association of Computational Methods in Engineering.

Shampine L, Reichelt M, Kierzenka J. (1999). Solving Index-1 DAEs in MATLAB and Simulink. SIAM Review 18(3):538-552.

The MathWorks Inc. (2019). MATLAB R2019a.

Tsai M, Wu S, Chang K, Lee G. (2007) Shaking table tests of a scaled bridge model with rolling-type seismic isolation bearings. Engineering Structures 29:694-702.

Walters M. (2015). Seismic isolation: The gold standard of seismic protection. In: Structural Performance, Structure Magazine.

Wang S, Hwang J, Chang K, Shiau C, Lin W, Tsai M, Hong J, Yang Y. (2014). Sloped multi-roller isolation devices for seismic protection of equipment and facilities. Earthquake Engineering \& Structural Dynamics 43:1443-1461.

Wang S, Lin W, Yang C, Chang Y K Huang, Hwang J, Hwang J. (2017). Recent progress in Taiwan on seismic isolation, energy dissipation, and active vibration control. In: 15th World Conference on Seismic Isolation, Energy Dissipation, and Active Vibration Control of Structures, New Zealand Society for Earthquake Engineering, Anti-seismic Systems International Society.

Wang S, Yu C, Cho C, Hwang J. (2019). Effects of design and seismic parameters on horizontal displacement responses of sloped rolling-type seismic isolators. Structural Control and Health Monitoring 26(5):e2342.

Wang S, Sung Y, Yang C, Lin W, Yu C. (2020). Control performances of friction pendulum and sloped rolling-type bearings designed with single parameters. Applied Sciences 10(20):7200.

Zhang C, Ali A. (2021). The advancement of seismic isolation and energy dissipation mechanisms based on friction. Soil Dynamics and Earthquake Engineering 146:106746. 